\title{
Calculated studies of the vibrational properties of the mode parameter of the electric power system containing asynchronous turbogenerators by their frequency characteristics
}

\author{
Yunus Bobojonov ${ }^{1}$, Bayram Seytmuratov ${ }^{1}$, A N Sultanov ${ }^{4}$, Bayram Fayzullaev ${ }^{2}$, Husanov Shahobiddin Hayrullo oglu ${ }^{3}$ \\ ${ }^{1}$ Karakalpak State University, Nukus, Uzbekistan \\ ${ }^{2}$ Nukus branch of Tashkent University of Information Technologies named after Muhammad Al-Khwarizmi \\ ${ }^{3}$ Department of "Electrical Engineering and Electrical Mechanics" Almalyk branch of Tashkent State Technical University \\ ${ }^{4}$ Tashkent State Technical University
}

\begin{abstract}
The article presents the results of a study of the oscillatory properties of the operating parameter of electric power systems during joint operation of synchronous and asynchronous turbine generators at the station and the influence of the proportional ratios of their powers on the oscillatory properties based on their amplitudefrequency characteristics.
\end{abstract}

\section{Introduction}

Low-frequency fluctuations of operating parameters in the range of $0.1-3.0 \mathrm{~Hz}$ are one of the main problems of reliability and functioning of power systems all over the world. This is due to the fact that the presence of such fluctuations can significantly reduce the permissible flows in the system and, as a result, worsen the economy while ensuring the required level of reliability [1-5].

The conducted studies of the modes of power systems show the expediency of the layout of power plants with a mixed composition of synchronous and asynchronous generators, which improves the operating conditions of synchronous generators, since the reactive power available by them will be used.. As studies show, in this case, the stability level of the synchronous turbine generator (STG) increases, its reliability and the technical and economic indicators of the station and the system as a whole improve. Also, the widespread introduction of static sources of reactive power will make it possible to create long-distance $\mathrm{AC}$ transmission lines with a station at the starting end, entirely consisting of an asynchronous turbine generator (ATG). Based on the results of previous studies, it is known that the indisputable advantages of the proposed power transmission are: the absence of the problem of dynamic stability in its traditional sense, practically unlimited length, the ability to take off power at any point in the line. The modes of modern power systems, the observed trend and new technical means for the generation and distribution of reactive power solve the problem of covering the reactive power of asynchronous generators and open up prospects for the creation of long- distance and ultra-long-distance AC power transmissions.

The studies were carried out according to the simplified Park-Gorev equations in the d-q coordinate system, by the method of small oscillations.

As you know, in the study of weakly damped free vibrations or forced vibrations under the action of periodic disturbing forces (asynchronous movement or a sharply variable periodic load), periodic solutions of the original equations are sought.

In the study of small oscillations, periodic solutions of linearized differential equations written in operator form are sought. One of the common methods for studying the dynamic properties of EPS - the frequency method - is also based on the operator form of linearized equations, in which the properties of the system in transient processes are determined based on the frequency characteristics of the operating parameters of the system.

\section{The mathematical statement of the problem}

The computational studies of the vibrational properties of the EPS were carried out according to the schematic diagram shown in Fig. 1.

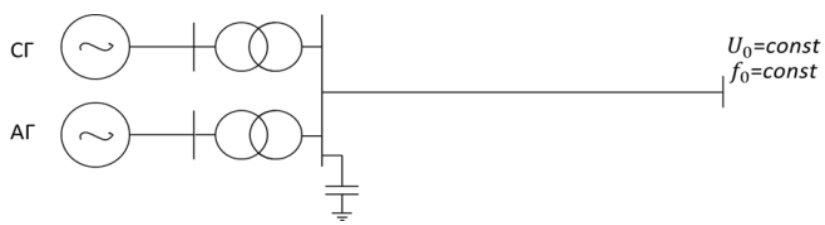

Fig. 1. Scheme of joint work of STG and ATG 
When STG and ATG work together at one station, the active power of the generators at the starting end of the transmission line in the initial mode is determined by the ratios:

$$
\begin{aligned}
& P_{L}=P_{S}+P_{A}-P_{1} \\
& P_{S}=(1-K) \quad\left(P_{L}-P_{1}\right) \\
& P_{A}=K\left(P_{L}-P_{1}\right)
\end{aligned}
$$

Where: $\mathrm{P}_{\mathrm{L}^{-}}$transmitted active power at the beginning of the line;

$\mathrm{P}_{\mathrm{S}}, \mathrm{P}_{\mathrm{A}^{-}}$active power of synchronous and asynchronous generators, respectively;

$\mathrm{K}$ - power ratio between STG and ATG.

When studying the oscillatory properties of the equations of transient processes of the stator of the STG and ATG, the static elements of the system are simplified by eliminating the transformer e. d. s and emf slip, active resistances of the stator of generators are also not taken into account [6-14].

The components of the voltage of the buses of infinite power (BWM) in this case can be determined by the total angle of power transmission:

$$
\begin{aligned}
& U_{d o}=U_{o} \sin \delta_{o} \\
& U_{q o}=U_{o} \cos \delta_{o}
\end{aligned}
$$

Where: $\delta_{o}=\delta_{g}+\delta_{s}$

In this case, the balance equation for the component currents is as follows:

$$
\begin{aligned}
& \mathrm{i}_{d l}=\mathrm{i}_{d c g}+\mathrm{i}_{d a g}-\mathrm{i}_{d c} \\
& \mathrm{i}_{q l}=\mathrm{i}_{q c g}+\mathrm{i}_{q a g}-\mathrm{i}_{q c}
\end{aligned}
$$

Where $i_{d c}, i_{\mathrm{qc}}-$ components of the power line charging power currents.

The linearized equations of the EPS elements, taking into account the simplification, have the form:

$$
\begin{aligned}
& \Delta U_{d g}=-\Delta \psi_{q} \\
& \Delta U_{q}=\Delta \psi_{d} \\
& \Delta \psi_{d}=G(P) \Delta U_{f}-X_{d}(P) \Delta \mathrm{i}_{d} \\
& \Delta \psi_{q}=-X_{q}(P) \Delta \mathrm{i}_{q} \\
& T_{j s} P^{2} \Delta \delta=-\psi_{d} \Delta \mathrm{i}_{q}-\mathrm{i}_{q} \Delta \psi_{d}+\psi_{q} \Delta \mathrm{i}_{d}+\mathrm{i}_{d} \psi_{q} \\
& \Delta U_{d g}=-\Delta \psi_{q A G} \\
& \Delta U_{q g}=\Delta \psi_{d A G} \\
& \Delta \psi_{d A G}=X_{m} \Delta \mathrm{i}_{d r}-X_{S} \Delta \mathrm{i}_{d A G} \\
& \Delta \psi_{q A G}=X_{m} \Delta \mathrm{i}_{d r}-X_{S} \Delta \mathrm{i}_{q A G} \\
& P \Delta \psi_{d r}=-S_{A G} \Delta \psi_{a r}-\Delta S_{A G}-R_{r} \Delta \mathrm{i}_{d r} \\
& P \Delta \psi_{q r}=S_{A G} \Delta \psi_{a r}+\Delta S_{A G}-R_{r} \Delta \mathrm{i}_{q r} \\
& \Delta \psi_{d r}=X_{r} \Delta \mathrm{i}_{d r}-X_{m} \Delta \mathrm{i}_{d A G} \\
& \Delta \psi_{q r}=X_{r} \Delta \mathrm{i}_{q r}-X_{m} \Delta \mathrm{i}_{d A G} \\
& T_{j A G} p \Delta \mathrm{S}_{A G}+X_{m} \mathrm{i}_{q A G} \Delta \mathrm{i}_{d r}-X_{m} \mathrm{i}_{d A G} \Delta \mathrm{i}_{q r}-X_{m} \mathrm{i}_{q r} \Delta \mathrm{i}_{d A G} \\
& +X_{m} \mathrm{i}_{d r} \Delta \mathrm{i}_{q A G}=0 \\
& \Delta U_{d g}=U_{o} \cos \delta_{o} \Delta \delta-X_{l} \Delta \mathrm{i}_{q l}+R_{l} \Delta \mathrm{i}_{d l} \\
& \Delta U_{q g}=-U_{o} \sin \delta_{o} \Delta \delta-X_{l} \Delta \mathrm{i}_{d l}+R_{l} \Delta \mathrm{i}_{q l} \\
& \Delta \mathrm{i}_{d l}=\Delta \mathrm{i}_{d c g}+\Delta \mathrm{i}_{d a g}-\Delta \mathrm{i}_{d c} \\
& \Delta \mathrm{i}_{q l}=\Delta \mathrm{i}_{q c g}+\Delta \mathrm{i}_{q a g}-\Delta \mathrm{i}_{q c}
\end{aligned}
$$

In the above equations, it is denoted: $U_{d}, U_{q}, \psi_{d c}$, $\psi_{q c}, \psi_{d A}, \psi_{q A^{-}}$longitudinal and transverse components of voltage and stator flux links of synchronous and asynchronous generators;

$\mathrm{T}_{\mathrm{jc}}, \mathrm{T}_{\mathrm{jAG}}$-inertial machine constants; $\delta, S_{A G}-$ the load angle of the synchronous generator and the slip of the asynchronous generator, taken positive in the generator mode.

Where: $G(p), X_{d}(p)$ and $X_{q}(p)$ - respectively, the operator conductivity and the operator resistance along the STG axes, taking into account three damping windings.

Transforming the above equations and including here the ARV s.d STG equations, we obtain:

$$
\begin{gathered}
\Delta U_{d g}=X_{q}(P) \Delta \mathrm{i}_{q c g} \\
\Delta U_{q g}=G(P) \Delta U_{f} X_{d}(P) \Delta \mathrm{i}_{d c g} \\
P^{2} \Delta \delta-\left(\psi_{q}+\mathrm{i}_{q} X_{d}(P)\right) \Delta \mathrm{i}_{d}+\left(\psi_{d}+\mathrm{i}_{d} X_{q}(P)\right) \Delta \mathrm{i}_{q} \\
+\mathrm{i}_{q} G(P) \Delta U_{f}=0 \\
\Delta U_{d g}+X_{m} / X_{r} \Delta \psi_{q r}+\left(X_{m}^{2} / X_{r}-X_{S}\right) \Delta \mathrm{i}_{q a g}=0 \\
\Delta U_{q g}-X_{m} / X_{r} \Delta \psi_{d r}-\left(X_{m}^{2} / X_{r}-X_{S}\right) \Delta \mathrm{i}_{d a g}=0 \\
\left(R_{r} / X_{r}+P\right) \Delta \psi_{d r}+\psi_{q r} \Delta S_{A G}+S_{A G} \Delta \psi_{q r}+R_{r} X_{m} / \\
X_{r} \Delta \mathrm{i}_{d a g}=0 \quad \\
\left(R_{r} / X_{r}+P\right) \Delta \psi_{q r}-\psi_{d r} \Delta S_{A G}-S_{A G} \Delta \psi_{d r}+R_{r} X_{m} / X_{r} \Delta \mathrm{i}_{q a g} \\
=0 \\
T_{j A G} P \Delta \mathrm{S}_{A G}-\left(X_{m} \psi_{q r} / X_{r}\right) \Delta \mathrm{i}_{d a g}+\left(X_{m} \psi_{d r} / X_{r}\right) \Delta \mathrm{i}_{q a g} \\
+\left(X_{m} \mathrm{i}_{q A G} / X_{r}\right) \Delta \psi_{d r} \\
-\left(X_{m} \mathrm{i}_{d A G} / X_{r}\right) \Delta \psi_{q r}=0 \\
\left(X_{l} / X_{s \ni}-1\right) \Delta U_{d g}+R_{l} / X_{s \ni} \Delta U_{q g}+R_{l} \Delta \mathrm{i}_{d c g}-X_{l} \Delta \mathrm{i}_{q c g} \\
+R_{l} \Delta \mathrm{i}_{d a g} X_{l} \Delta \mathrm{i}_{q a g} U_{0} \cos \delta_{0} \Delta \delta=0 \\
-R_{l} / X_{s \ni} \Delta U_{d g}+\left(X_{l} / X_{c \ni}-1\right) \Delta U_{q g}+X_{l} \Delta \mathrm{i}_{d c g}+R_{l} \Delta \mathrm{i}_{q c g} \\
+R_{l} \Delta \mathrm{i}_{q a g}+X_{d a g} \Delta \mathrm{i}_{d a g}-U_{0} \sin \delta_{0} \Delta \delta=0 \\
\Delta U_{f}=\Sigma W_{A P B}(P) /\left(1+P T_{\theta}\right) \Delta P
\end{gathered}
$$

Here the first three equations describe the transient process in synchronous, the next five - in asynchronous generators, the rest - in the line, as well as in the ARV system, and its transfer function is represented in the form: $W_{P}(P)=\Delta U_{f} / \Delta P=\left(K_{O P}+P K_{1 P} /\left(1+P T_{1}\right)+\right.$ $\left.P^{2} K_{2 P} /\left(1+P T_{2}\right)\right) /\left(1+P T_{\theta}\right)$.

where P- mode parameter (U, I, f, $\delta$ and etc)

$T_{\theta}, T_{1}, T_{2}$-time constants, respectively, of the STG pathogen, differentiating and doubly differentiating ARV elements;

$K_{O P}, K_{1 P}, K_{2 P}$-amplification factors, respectively, by deviation $(\Delta \mathrm{P})$ the first $(\mathrm{pP})$ and the second $\left(\mathrm{p}^{2} \mathrm{P}\right)$ derivatives of the controlled parameter $\mathrm{P}$. Obtaining the characteristic equation of the system under study in the form of a polynomial in $\mathrm{p}$ by expanding the determinant, or, which is the same, excluding all unknowns from the system of equations one by one, presents special difficulties [15-20].

From the point of view of using computer programs, it is most desirable to represent the characteristic equation in the form of a determinant, the order of which is equal to the number of equations in the system. This representation of the characteristic equation allows the use of the frequency method.

The presented system of equations makes it possible to investigate the oscillatory properties of the system under 
consideration when regulating the emf of the STG as a function of various operating parameters (respectively, in terms of voltage and stator current, total angle of power transmission) STG and (in terms of stator current and slip) ATG and other dynamic characteristics of the system. The characteristic determinant of the system of equations for the total angle of STG power transmission has the form:

$$
F(P)=W_{\delta}(P) /\left(1+P T_{\theta}\right)
$$

where: $\mathrm{F}(\mathrm{P})$ - transfer functions of the ARV system, reflecting the regulation of e. with STG according to the total angle of power transmission $(\delta)$.

Denoting the characteristic determinant of the system under study through the operator expression $A_{i j}(p)$ :

$\mathrm{D}(\mathrm{P})=\left[\mathrm{A}_{\mathrm{ij}}(\mathrm{P})\right]$

where $A_{i j}(p)$ - elements of the characteristic determinant depending on the parameters of the EPS mode.

To find the expression of the transfer function of the ARV along the transmission angle channel $(\delta)$, the characteristic equation has the form:

$D(P)=H(P) D_{1}(P)-D_{2}(P)=0$

$$
\Delta U_{f} / \Delta \delta=Y(P)
$$

where: $D_{1}(P), D_{2}(P)$-minors of the characteristic determinant.

$\mathrm{Y}(\mathrm{P})$ - a complex expression that includes transfer functions and parameters of all other elements - external in relation to the ARV system under consideration and the values characterizing its initial steady state.

The structural diagram of the studied EPS is a closed circuit consisting of ARV elements and an external circuit in relation to the ARV.

$W_{E X}(j \omega)=\Delta \delta / \Delta U_{f}=1 / Y(j \omega)$;-external

frequency response.

The physical explanation of the frequency characteristics of the external system and ARV can be given using the structural diagram shown in Fig. 2, obtained by opening the closed system at the ARV input$$
\text { I }
$$

\begin{tabular}{l|l|l|l|l}
$\Delta P_{\text {in }}$ & \multicolumn{3}{|c}{$\Delta U_{f}$} \\
& $W_{\text {out }}(j \omega)$ & & $W_{E X}(j \omega)$ &
\end{tabular}

Fig. 2. Open circuit of the elements of the studied EPS. Where: $W_{A R V}(j \omega)=\Delta U_{f} / \Delta P_{i n^{-}}$frequency response of ARV.

$$
W_{E X}(j \omega)=\Delta P_{\text {out }} / \Delta U_{f} \text {-external system frequency }
$$
response.

$W_{\text {time }}(j \omega)=W_{A R V}(j \omega) W_{E X}(j \omega)=\Delta P_{\text {out }} / \Delta P_{\text {in }}$-frequency response of an open system. A periodic signal is applied to the input of link I: $\Delta P_{i n}(j \omega)=\left|\Delta P_{i n}\right| e^{j w j+\mathrm{v}}$

Output signal: $\Delta U_{f}(j \omega)=\left|\Delta P_{i n}\right| e^{j w j+\mathrm{v}} \quad$ undergoes amplitude and phase changes. The phase shift between the $\mathrm{V}_{\mathrm{ARV}}$ output and input signals is a function of frequency. The output signal of link I is fed to the excitation winding of the STG of the external system - ink II, where the input signal also undergoes an amplitude and phase change. Phase shift between output $\Delta P_{\text {out }}$ and input $\Delta P_{\text {in }}$ signals:

$\mathrm{F}(\mathrm{j} \omega)=V_{A R V}(j \omega)+\varphi_{\text {rot }}(j \omega)$

where: $\varphi_{\text {rot }}(j \omega)=\varphi_{\text {rot.sg }}(j \omega)+\varphi_{\text {rot.ag }}(j \omega)$-phase shift in a link II.
Thus, the open-circuit frequency response of the system under study has the form:

$$
\begin{gathered}
W_{\text {time }}(j \omega)=W_{A R V}(j \omega) W_{E X}(j \omega)=\left[\left(\mathrm{K}_{0 P}+j \omega \mathrm{K}_{1 P} /(1+\right.\right. \\
\left.\left.\left.j \omega \mathrm{T}_{1}\right)-\omega^{2} \mathrm{~K}_{2 P} /\left(1+j \omega \mathrm{T}_{2}\right)\right) /\left(1+j \omega \mathrm{T}_{\theta}\right)\right] W_{E X}(j \omega)= \\
A e^{j F w} .
\end{gathered}
$$

Below are the results of computational studies of the amplitude-frequency characteristics of the total power transmission angle of EPS when the composition of the station changes from the traditional layout $(K=0)$ to the complete replacement of all STGs with ATGs $(K=1)$, each time increasing by $20 \%$ ( Fig. 3).

In the proposed plant layouts, the required reactive power for the ATG is generated by jointly operating STGs and partially by the transmission line, i.e. there is no need to install additional reactive power sources.

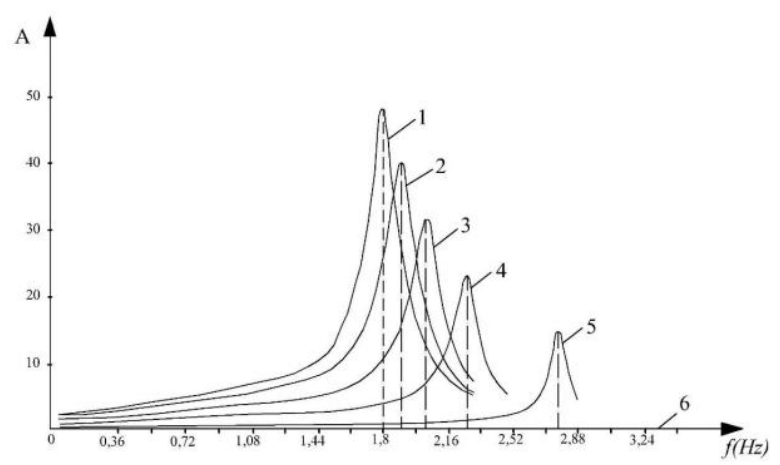

Fig. 3. Amplitude-frequency characteristics of EPS, in the presence of ATG in percentage ratios. $1-0 \%$; 2-20\%; 3-40\%; 4-60\%; 5-80\%; 6-100\%;

As can be seen from the characteristics, with an increase in the share of ATG in the transmission of power through the power transmission line while maintaining the unchanged installed power of the station, the damping of electromechanical vibrations increases, and with a complete replacement of the STG with ATG, electromechanical vibrations are almost absent.

The increase in vibration damping due to the ATG is explained, firstly, by the influence of the damping properties of the ATG, and secondly, by an increase in the reactive power generated by the jointly operating STGs and, accordingly, their EMF [21-23].

From the obtained amplitude-frequency characteristics, it should be noted that with the introduction of ATG at the station, the resonant frequency shifts upward, for example, the resonance frequency value at $\mathrm{K}=1$ is approximately two times greater than at $\mathrm{K}=0$.

Such a shift of the resonant frequency of the EPS operating parameters from the possible frequency bandwidth allows preventing system accidents caused by low-frequency weakly damped oscillations.

\section{Conclusions}

Thus, the joint layout of the station from STG and ATG not only improves the operating and technical and economic indicators of the power plant, but also creates a natural damping of fluctuations in operating parameters, and this layout also improves the stability of the STG, the advantage 
is manifested especially in transient modes, operating in parallel. STG and ATG act as a self-regulated system of fluctuations of the mode parameters, localized within the station buses, and the EPS will have a greater attenuation than a system containing only STG.

\section{References}

1. R.Liu, Y.Liao " Damping characteristics analysis for asynchronized turbo-generators and asynchronous turbogenerator with pss". April 2009 Zhongguo Dianji Gongcheng Xuebao/Proceedings of the Chinese Society of Electrical Engineering 29(12):93-98

2. "Mathematical modeling and dynamic simulation of a class of drive systems with permanent magnet synchronous motors", m. Mikhov, applied and computational mechanics 3 (2009) 331-338.

3. R. Lateb, J. Enon, and L. Durantay, "High speed, High power electrical induction motor technologies for integrated compressors" in Proc. International Conference on Electrical Machines and Systems, ICEMS '09, November 2009, pp. $1438-1442$.

4. T. Wang, F. Wang, H. Bai and J. Xing, "Optimization Design of Rotor Structure for High Speed Permanent Magnet Machines," in Proc. IEEE Intl. Conference on Electrical Machines and Systems, ICEMS'07, Oct. 2007, pp. 14381442.

5. I.Rakhmonov, A.Berdishev, N.Niyozov, A.Muratov and U.Khaliknazarov. Development of a scheme for generating the predicted value of specific electricity consumption // CONMECHYDRO - 2020. IOP Conf. Series: Materials Science and Engineering. $883 \quad$ (2020) 012103. doi:10.1088/1757-899X/883/1/012103

6. F.A.Hoshimov, I.I.Bakhadirov, M.S.Kurbanbayeva, N.A.Aytbayev. Development of specific standards of energy consumption by types of produced products of the spinning product // RSES 2020. E3S Web of Conferences. 216 (2020) 01169. https://doi.org/10.1051/e3sconf/202021601169

7. F.A.Hoshimov, I.I.Bakhadirov, A.A.Alimov, M.T.Erejepov. Forecasting the electric consumption of objects using artificial neural networks // E3S Web of Conferences. 216 (2020) 01170.

8. I.U.Rakhmonov, F.A.Hoshimov. Development of an algorithm for evaluating the dominant factors that have the greatest impact on the energy intensity of products // ENERGY-21. E3S Web of Conferences. 209 (2020) 07018. https://doi.org/10.1051/e3sconf/202020907018

9. Usmanov E.G. Stability in a parallel resonant circuit with active load // RSES 2020. E3S Web of Conferences. 216 (2020) 01160. https://doi.org/10.1051/e3sconf/202021601160 10. Usmanov E.G., Khusanov B.M. Phase relations in resonant circuits with a wide falling section on the amplitude characteristic // RSES 2020. E3S Web of Conferences. 216 (2020) 01161. https://doi.org/10.1051/e3sconf/202021601161 11. I.U.Rakhmonov, K.M.Reymov and S.H.Dustova. Improvements in industrial energy rationing methods // MIP: Engineering-2020. E3S Web of Conferences. 862 (2020) 062070. doi:10.1088/1757-899X/862/6/062070

12. I.U.Rakhmonov, K.M.Reymov. Statistical models of renewable energy intermittency // RSES 2020. E3S Web of
Conferences. $\quad 216$

01167. https://doi.org/10.1051/e3sconf/202021601167

13. I.U.Rakhmonov, N.N.Kurbonov. Analysis of automated software for monitoring energy consumption and efficiency of industrial enterprises // E3S Web of Conferences. 216 (2020) 01178. https://doi.org/10.1051/e3sconf/202021601178 14. F.A.Hoshimov, I.U.Rakhmonov, N.N.Niyozov. Technology to reduce energy costs in the electric steel melting shop // ENERGY-21. E3S Web of Conferences. 209 (2020) 07017. https://doi.org/10.1051/e3sconf/202020907017 15. I.Bakhadirov, N.Markaev, G.Aslanova, R.Tanatarov, S.Makhmuthonov. Differentiated tariffs of electricity for the improvement of steelmaking Uzbekistan // CONMECHYDRO - 2020. IOP Conf. Series: Materials Science and Engineering. $883 \quad$ (2020) 012121. doi:10.1088/1757-899X/883/1/012121

16. A.D.Taslimov. Selection of a complex of parameters of distribution electric networks with respect to technical limitations // ENERGY-21. E3S Web of Conferences. 209 (2020) 07013. https://doi.org/10.1051/e3sconf/202020907013 17. Olimjon Toirov, Allabergan Bekishev, Sardor Urakov and Utkir Mirkhonov E3S Web of Conferences 216, 01116 (2020), https://doi.org/10.1051/e3sconf/202021601116

18. Olimjon Toirov,Kamoliddin Alimkhodjaev, Nurali Pirmatov and Aziza Kholbutaeva E3S Web of Conferences 216, 01119

(2020), https://doi.org/10.1051/e3sconf/202021601119

19. K.M.Reymov, G.Turmanova, S.Makhmuthonov, B.Uzakov. Mathematical models and algorithms of optimal load management of electrical consumers // E3S Web of $\begin{array}{llll}\text { Conf. } & 216 & \text { (2020) } & 01166 .\end{array}$ https://doi.org/10.1051/e3sconf/202021601166

20. A.N.Rasulov, M.R.Ruzinazarov, N.Toirova, T.Sh.Alibekova. Graphical-analytical method for constructing load characteristics // RSES 2020. E3S Web of Conferences. $216 \quad 01171$. https://doi.org/10.1051/e3sconf/202021601171

21. Yu.Bobozhonov, B.Seytmuratov, B.Fayzullaev, A.Sultonov. Study of the influence of different designs of massive rotor of asynchronous generator on their maximum power // RSES 2020. E3S Web of Conferences. 216 (2020) 01177. https://doi.org/10.1051/e3sconf/202021601177

22. Rakhmonov I U, Reymov K M, Najimova A M, Uzakov B T and Seytmuratov BT 2019 Analysis and calculation of optimum parameters of electric arc furnace Journal of Physics: Conference Series 1399 doi:10.1088/17426596/1399/5/055048

23. Yu.M.Bobozhonov, K.M.Reymov, B.T.Seytmuratov, T.Kh.Khakimov. Research of the dependence of the resistance of asynchronous generators with massive rotors on their design // RSES 2020. E3S Web of Conferences. 216 (2020) 01168. https://doi.org/10.1051/e3sconf/202021601168 